\title{
Gamified Solutions in Healthcare - Testing Rehabilitation Games in Finland and Asia
}

\author{
C. Kattimeri ${ }^{1}$, P. Qvist ${ }^{1}$, N. Katajapuu ${ }^{1}$, P. Pitkäkangas ${ }^{1}$, H. \\ Malmivirta $^{1}$, M. Luimula ${ }^{1}$, A. Pyae ${ }^{2}$, T.N. Liukkonen ${ }^{2}$, and J. Smed ${ }^{2}$ \\ ${ }^{1}$ Turku University of Applied Sciences, Turku Game Lab \\ Joukahaisenkatu 3, 20520 Turku, Finland \\ Phone: +358403550839 \\ e-mail: mika.luimula@turkuamk.fi \\ ${ }^{2}$ University of Turku, Department of Information Technology \\ FI-20014 University of Turku, Finland
}

Abstract: This paper presents a comprehensive summary of the Gamified Solutions in Healthcare (GSH) research project, which is a joint research project between Turku University of Applied Sciences and the University of Turku. The goal of the project is to promote exercise, social inclusiveness and enhance quality of life, aiming at developing new services and effective activity solutions for the elderly through gamification. During the research project elderly people were included in the development and testing of games so that they could be used for more than just entertainment purposes. According to all of our tests elderly enjoy playing exergames, and digital games can be an effective way to enhance the quality of life of the elderly. In the case studies it was observed that the players where motivated while playing but motivation should also be maintained throughout the gameplay. The elderly gave overall positive feedback for the idea of using digital activity games for exercising.

Keywords: Gamification, Serious Games, Rehabilitation, Usability, Field Experiments

\section{Introduction}

According to the World Health Organization active ageing is the process of optimizing opportunities for health, participation and security in order to enhance quality of life as people age. Seniors are easily alienated from the society due to the digital divide and unequal opportunities of using modern technology; therefore it is very important to include them in the development of the information society [1].

In the industrialized and developed countries, the growth of both number and proportion of the elderly in the population is evident, and the elderly is one of the most rapidly growing age groups [2]. The age-related decline of physical and cognitive capabilities for an individual can present significant impact on the quality of life and 
furthermore elevate the need for additional social and health related services. These two factors combined can lead to rising healthcare and wellbeing related costs on the individual and societal levels. Providing physical exercise to the muscles [3] and cognitive and memory related exercise to the brains [4] can alleviate, slow down, reverse and even stop this decline $[5,6]$.

Nowadays, the use of digital games is not only restricted for the purpose of entertainment, but expanded in areas such as education, healthcare, business and the military as well. Digital games are designed to make the players experience high levels of motivation and engagement in the game itself. One of the key motivators for starting to further study the gamified methods to apply for healthcare was to employ this high motivational level and engagement found within digital games in useful and productive application areas. These games designed for other primary purposes than solely for entertainment purpose are generally defined as serious games. Furthermore, gamification is the term used for the process of applying game-like thinking and game mechanics to traditionally non-game applications and functions (e.g. education and exercise) to make them more fun and engaging [1].

This paper is structured to present the Gamified Solutions in Healthcare research project, and consequentially the serious games developed within this research project, and furthermore discussing the findings and results of various studies and tests conducted during the timeframe of the research project. The developed games and the relevant tests are presented as case studies, and a literature review on motivational factors for elderly stroke patients is also included. The paper concludes with the summary of various tests conducted during the research project, presenting a summary of the impact of these tests and a compilation of test groups with the number of test subjects involved in the studies from all the countries participated in the tests of the research project.

\section{Gamified Solutions in Healthcare}

Gamified Solutions in Healthcare (GSH) is a joint research project between Turku University of Applied Sciences and the University of Turku. In the course of this project, new gamified services are researched and developed in cooperation with Serious Games Finland Oy, Attendo Finland Oy, City of Turku Welfare Division and Puuha Group Oy. The goal of the project is to promote exercise, social inclusiveness and enhance quality of life. GSH is funded by Tekes, the Finnish Funding Agency for Innovation [1].

The GSH project aims at developing new services and effective activity solutions for the elderly through gamification. The aim of the GSH project is to include elderly people in the development and testing of games so that they could be used for more than just entertainment purposes.

In the course of the GSH project we will conduct more research about the attitudes of the seniors towards game-playing and digital games in general. We need more focus group interviews as well as some usability testing with console games. The research requires systematic analysis about the existing games for seniors, and the attitudes and perceptions of health and social care workers that work with seniors. In summary, our research topics include: gamification mechanisms, usability for elderly people, the 
effectiveness of gamified solutions for elderly people (e.g. business and production models), and attitudes and acceptance of games by the elderly people [1].

The Finnish game development industry needs new solutions for active health ageing and, therefore, cooperation with Asian partners will open up new possibilities to build innovations and generate new business opportunities. Researchers around the world are now activating even if this research field is still quite new [1]. Dealing with the ageing population is a challenge not only in Asian societies but also in Western societies such as Finland. Therefore, research organizations are very active in the field of serious game development and developing games for elderly. Thus, we believe that these kinds of rehabilitation concepts will be needed in the future $[7,8]$.

One of the initial goals of the GSH project was to design and implement a Virtual Nursing Home (VNH) in collaboration with our industrial partners Puuha Group, GoodLife Technology, City of Turku and Attendo Oy in Finland. VNH is a concept that provides alternative solutions for the elderly's self-management and self-care, and prevents them from social isolation while, at the same time, reduces the workload of the healthcare professionals. The methodologies used for the implementation of this project were User-Centered Design (UCD) and rapid prototyping [9].

\section{Case Studies in GSH Project}

In this section we present six studies we have conducted related to the usability of gamification in encouraging physical or mental activity. Although most of these studies take different approaches and have different test groups, they reveal features that are common to physical activity oriented gamification. In addition, we present a literature review on motivational factors for elderly stroke patients.

\subsection{Pre-study: Testing Glider game in Japan}

In 2013, we focused on the usability evaluation of a prototype game called "The Glider", which is a Kinect-based game by Serious Games Finland (SGF). The study identifies difficulties, and suggests improvements related to the user interface development of "The Glider". The controlling motions in the game have been developed in close cooperation with medical doctors, physiotherapists, and patients in the terms of well-being and light exercise. This study utilizes methods based on Kansei Engineering. The Japanese word "Kansei" can be interpreted as emotion, feeling, receptivity, or sensibility. In essence, Kansei Engineering is a consumer-oriented technological research field that supports engineers in the development or improvement of products via evaluating the "Kansei" of the users [7,8].

We conducted usability tests with 12 test subjects ( 6 male and 6 female) who were all Japanese students. The test subjects played the prototype game and spoke aloud their thoughts while playing. Two video cameras were recording the test scene $[7,8]$.

The test results showed that the beginning of the game was very simple and easy to play having just one motion control per level. The players were learning the exercises while playing the first levels; however, they should receive more information and guidance before starting to play. Some of the motion controls that were developed for rehabilitation increased the complexity of the game. The players were motivated while playing the 
game. Moreover, difficulty levels and the understanding of the game seemed to be in balance. The players were interested to play and learn new things; however, after the first four levels they seemed to slightly lose their motivation. In these four levels, the players were iteratively reminded of how to control the glider (accelerating, and moving in all directions). This is needed later with more complex steering motions, although the next levels showed that the players were not able to control the left and right movement anymore. Generally speaking, the game flow seemed to be in balance between difficulty and usability from the beginning until the fourth level $[7,8]$.

\subsection{Case SportWall and commercial games}

We investigated the usability of two commercial games and a physical activity game developed by our project collaborator, Puuha Group, Finland. The main objectives of this pilot study were: to investigate the usability and usefulness of games for the elderly, to evaluate the usability and usefulness of multimodal input devices (Kinect, PlayMove, web-camera/Xtreme reality) for the elderly, and to understand the general user experiences of elderly in playing games $[1,9,10]$.

The games chosen for the tests were Puuha Group's SportWall (using Xtreme reality technology and a web-camera), Microsoft Xbox's Kinect-based climbing game and Playstation3's PlayMove tennis game. In the SportWall game the player uses particular body postures and gestures to control the roller-skating character in the game $[1,9,10]$.

Our test participants were two groups of elderly between the ages of $65-85$ years. The first group had 5 moderately active elderly, whereas the second group had 5 less active elderly but only 2 showed up for the tests. The second group had health problems and limited short-term memory and they could not participate in the tests or answer the questionnaires. Consequently, we only collected feedback from the moderately active elderly group and our results are based on that. We used two video cameras (front and back views) to record the actions and gameplay of the elderly. In addition we carried out questionnaire sessions that were captured by a voice recorder $[1,9,10]$.

In general, the elderly participants in our study were somewhat physically active and they did not have prior experience with digital games. In the test of the climbing game, the elderly had some problems in the beginning but after the tutorial and the guidance from the researchers they could manage without major challenges. Also, we found out that the user-interface, music and audio feedback in the game were not elderly-friendly. However, Kinect-based interaction was effective for the elderly. In the test of the tennis game, the elderly faced challenges with the buttons of the PlayMove controller. Nevertheless, they liked the idea of the tennis game. According to the test participants the last game, SportWall, was simple and had a clean interface. The interaction with the game was easy due to the use of a traditional webcam. However, some game actions (e.g. jump, sit) were unsafe for the elderly. In general, Kinect for the Xbox One was the most effective input device for the elderly yet the scores of the other two devices were not noticeably different $[1,9,10]$.

According to the interview with the caregiver of the service home, game-based physical activities are interesting and they can improve the motivation of the elderly in doing physical exercises. The socialization of the elderly can also be enhanced. The caregiver 
advised that the games should be simple and easy because of the elderly's limitations in mobility and memory $[1,9,10]$.

Furthermore, we conducted a pre-study that consisted of gathering and studying existing games for seniors, conducting a pre-test on console games and interviewing potential users of serious games. The search was limited to games that could enhance physical, mental or social well-being. In addition, the senior-friendly or unfriendly features of the discovered games were evaluated. Also, the barriers or attitudes of introducing games for the elderly were examined. The findings from the pre-studies revealed the limitations of the existing games and technologies. Not all the commercial games are accessible for the elderly. Additionally, most exergames do not support elderlyfriendly design and gameplay. The limitations of the current Kinect-based exercise games are lack of game customization, lack of effective feedback, and lack of long-term study. However, it was discovered that the existing games have potential in being reused with further modifications and enhancements in the future game development and testing within the GSH project. In general, the area of gamification for elderly and healthcare has noticeable challenges that need to be faced so that the researchers can investigate the benefits of digital games for elderly if any exist [10].

\subsection{Case Old Photos on Map}

The population in Europe is ageing dramatically and the ageing situation in Finland is even more challenging. Therefore, we propose "Old Photos on Map" (Vanhat kuvat) webapplication to activate the memory of the ageing population [11]. Malmivirta et al. have found that several researchers have come to the conclusion that cognitive mental activity is of high-importance for mental health. The effectiveness of using old photos to enhance brain health has been examined in the research and development project "Art and culture - Keys for better Brain Health" [12].

Malmivirta et al. advocate that in autobiographical activities like watching and sharing experiences of old photos, exploring punctum photographs allows an emotional connection to be formed with the most significant people, places and events in our lives. Subjective sense of time refers to the ability to shift to thinking about something that has happened in the past. Memory layers and visual perception are in active motion in this phase, when narratives, photographs and maps are integrated into each other. The autobiographical memory and discussions about the old photos require complicated cooperation between our cognitive and emotional processes which activate different parts of the brain. The brain feels well when the environment promotes activation and involves social interaction. The emergence of memory disorders can be delayed by keeping the brain occupied with new and complex tasks throughout our lives [11].

The aim of "Old Photos on Map" web application is to wake up peoples' memories and experiences from their childhood and earlier life experiences as well as personal hidden stories. The "Old Photos on Map" web application research and development work applied the practical action research strategy in which the main objective was to develop an application to activate cognitive memory health of the aged people. During the research and development process, any need for changes was recorded in line with the objectives and also the pedagogic plans with the interventions of the test groups. 
During the research and development process, the tests were conducted in two ways. Firstly we examined the design issues related to the user interface of the application. A virtual agent in the application encourages discussions between people in a group. We compared multiple agents and interface designs with different color variations and the final interface elements were chosen through questioning 24 Finnish people between 5693 years old. According to the results from the questionnaires larger text was preferable. The agent that was chosen was described as kind, adult, trustful and interesting [6].

Secondly, we studied the "Old Photos on Map" web application without the agent focusing more on the interventions with the group activities of the elderly people using means-ends art pedagogy and social cultural learning strategy. At the same time we are recording the changes that are needed for the application. We had three test settings with the collaboration of the third sector organizations. In the first two groups the participants did not have any diagnostic memory deceases (average age 30-75 years, $n=7$ ) and in the third group the members had all some kind of diagnosed memory problems (average age 55-80 years, $n=14$ ). The tests have been conducted during May and June 2016 and one more will be made on August 2016.

The first look at the data shows that the application "Old Photos on Map" activates the memory of the participants and brings out past memories. It also makes the participants to share their memories and learn more from each other's memories. It seems that when looking at the old photos on the map, diving into the punctum of the old photos and exploring the layers of the photos it allows an emotional connection to be formed with the most significant places, events and people in our lives. It also seems that a special pedagogical frame is needed for the interventions with different kinds of people and groups for revealing the hidden stories of the photos used. The test results seem to be very promising also with the people suffering with memory problems. While looking at old photos on the map a lot of sharp memories came out to be shared. The overall activity was growing, the eyes were sharpening and social interaction was richer.

\subsection{Case SportWall and PhysioWall}

We studied the usability and reception regarding SportWall (Game A) and PhysioWall (Game B) using SUS and GEQ questionnaires and interviews. In total we had 19 participants with overall average age $72 \pm 11$ years $(n=19)$. We had two testing settings: urban and rural. In the urban setting we had seven participants $(n=7,3$ female and 4 male), and in the second testing session in the rural setting we had $12(\mathrm{n}=12,7$ female and 5 male) participants. Age distribution was similar in both settings, and both genders were represented equally (10 female, 9 male) [13].

These games have been our first games that were tested with the participants that belong to our targeted age group. From these games we have learned what kind of things seem to work with our target group and what does not work. To remedy the problems we found during the testing, we are currently doing a redesign on both games. We had designed the games to have clear graphics without a screen clutter, which is seen on games aimed for younger players. Despite our efforts, the participants still had problems with knowing what they should avoid and what they could collect on the Game A. The graphics problem might be partially caused by the unfamiliarity of the activity represented in the game A as the interviews and comments by the elderlies during the gameplay revealed their 
confusion about these matters. The speed in which things happen on the Game A was also a problem, albeit the game was significantly slower than the original version which was designed for the younger players. The next version of the game will have its pacing further slowed down, and the context of the game will be more familiar to the target group [13].

Game B had originally a virtual instructor whose movements' the player should match, and a graph that showed what kind of movements will be coming after the current one. This caused confusion as some of the participants were not sure which one to follow or they missed the correct movements because the extra graph grabbed their attention. In Game B, players had to raise their hands several times above their heads. This was a problematic procedure for several elderly, and prevented them from completing the required movements. These problems are addressed in the new version of the game where e.g. the indicator for the upcoming motion has been removed [13].

In the future field tests, the gaming sessions will be longer as it was noted that during the short sessions the participants were aware about their surroundings and the observers. The longer sessions might help the participants to relax and immerse more on the game, instead of them being filling questionnaires in every turn after few minutes of the play. This might allow us to see more emotions rising from the gameplay experience [13].

SUS is a simple tool for measuring basic usability of a software-based artefact, and it proved to be usable and informative also with the elderly, mainly thanks to its brevity. GEQ in the other hand is a questionnaire which contains 50 or in the case of multiplayer situation 67 questions. The length of the GEQ brought up questions and comments from the participants during the testing sessions (e.g. "When will this questionnaire end?", "How many questions are left?"). In the future, the game experience research with elderly, or with other people who do not play games regularly or at all, would benefit from a shorter and more understandable questionnaire [13].

\subsection{Case Skiing Game}

We evaluated the usability of an exergame called "Skiing game" for Japanese elderly. We also investigated the elderly's engagement in playing the Skiing game. The study was conducted at a therapy training room at the Sendai City Health Promotion center in Japan. We recruited 24 elderly participants between the ages of 60 and 85 years. In the usability testing, the elderly participants played the Skiing game, which is a game- based Skiing activity, originally designed for the Finnish elderly. To play the game, the elderly participants moved their hands forward and backward as a conventional double-pole Skiing activity. A traditional webcam that uses Xtreme Reality technology was used to detect the player's movements in the game. A screenshot from the Skiing game can be seen in Figure 1. Before the participants started playing the game they were asked questions regarding their attitude towards physical exercise. After they have played the game they were asked questions about the usability of the game, their engagement in the gameplay, their motivation, and attitude towards game-based exercise [14]. 


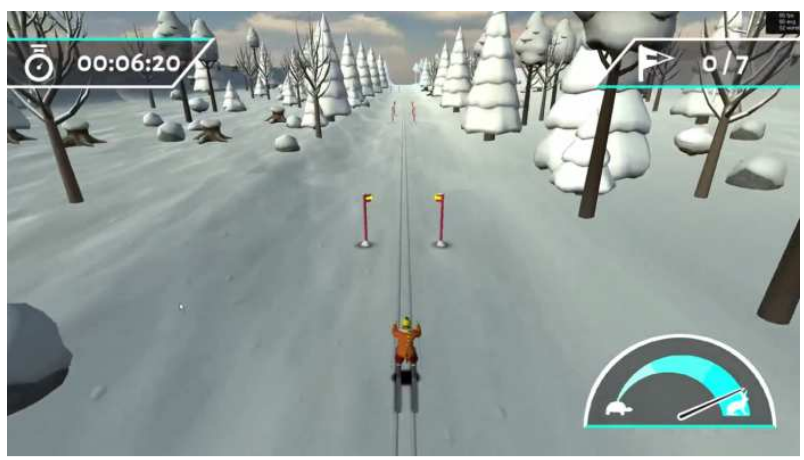

Figure 1 Screenshot from the Skiing Game

Based on the findings from the usability testing in Japan, we observed that the experiences and feedback of the elderly during and after the gameplay were noticeably positive. They were interested in playing the game and they would like to play it again. Therefore we claim that the Skiing game is an easy and user-friendly game for the Japanese elderly. Furthermore, most of the elderly agreed that playing digital games is an easy and effective way of exercising [14].

According to our observations from this study, we recommend the following design guidelines for usability practitioners. Firstly, we should take into consideration that the elderly should not be distracted from the gameplay therefore the game interface, context and contents should be simple and uncluttered. Secondly, excessive in-game instructions and unnecessary audio feedback should be avoided. Visual cues are important in the game, especially for novice elderly players. Controller-free and gesture-based interaction is effective for the elderly. Additionally, natural and familiar game actions can engage the elderly players more. To reduce the elderly's frustration while playing is always important, even if they did not achieve a particular task. Finally, while designing a game for elderly, it is very important to reduce and prevent the risk of falling [14].

In the course of the GSH project we tested and evaluated the usability of the Skiing game for Finnish elderly as well. The study was conducted at an elderly service home in Finland. We recruited 21 elderly participants, who were aged between 60 and 85 years. The results of the Finnish usability tests as well as a comparison between the Japanese and Finnish tests will be published in the near future.

\subsection{Case Brain Trainer Exercise Game}

This study was focusing on verifying the level (+ or -) of brain activity during the test subjects playing the "Brain Trainer Exercise Game" developed by Sendai Television, and furthermore evaluating the usability and acceptability of the game by observing the test subject behaviour during the gameplay. The test groups comprised of test subjects from Finnish and Japanese elderly population [15]. 


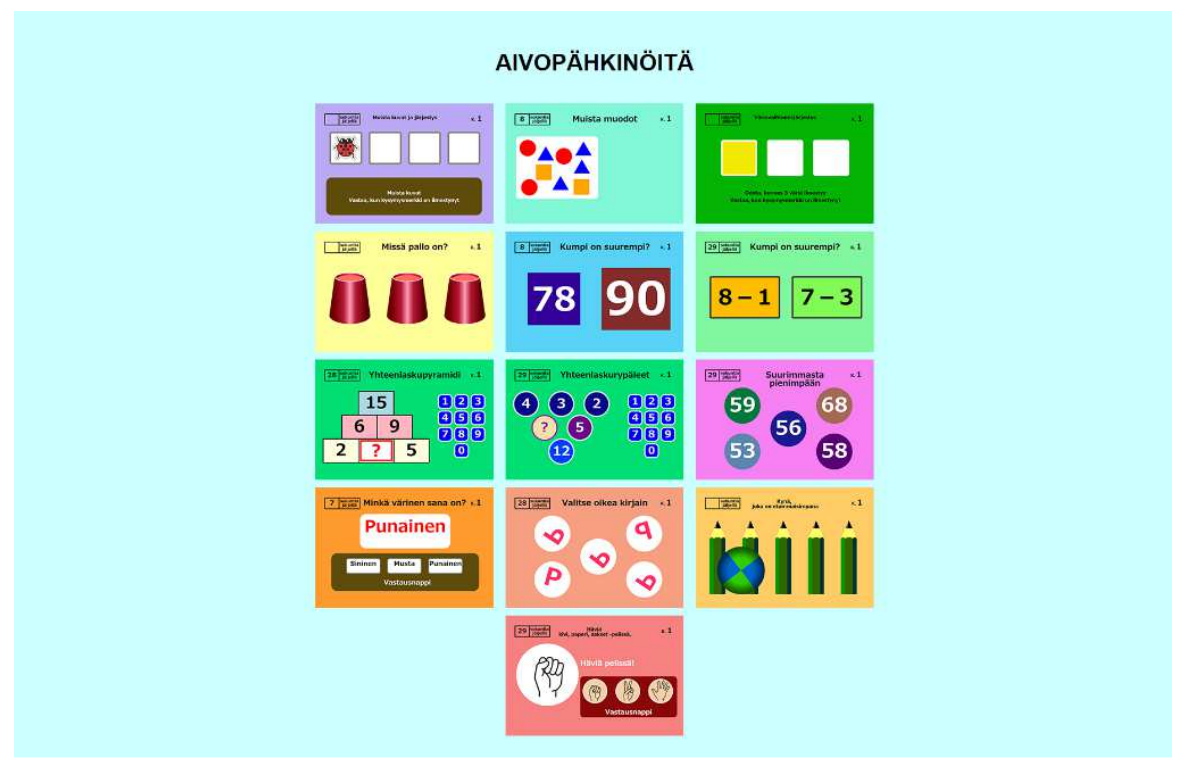

\section{Figure 2 Brain Trainer Exercise Games main menu localised in Finnish language showing all 13 minigames.}

The Brain Trainer Exercise Game was developed for the purpose of enhancing the cognitive skills of elderly population. The game includes 13 minigames shown in Figure 2 and one pen and paper calculation task. The main menu allows the player to freely choose their preferred minigame in any desired order. Each minigame has four different difficulty levels progressing from easiest $(*)$ towards the most difficult $(* * * *)$, with the difficulty being derived from different parameters depending on the theme of the minigame. These varying difficulty parameters can consist of for example limited time for the task within the minigame or number of objects appearing during the minigame for cognitive tasks such as memory exercise. Varying the difficulty parameters allows the game to provide more challenging tasks for cognitive skills related to perception or requiring more speed from the player to provide the correct answers to the given tasks. The thirteen minigames focus on different areas of cognitive and memory skills, with themes ranging from numeric calculations to remembering different colors and shapes, and following the moving objects and afterwards remembering different tasks. The Brain Trainer Exercise Game was designed to be played with easy-to-use user interface with a device that employs a touch screen. The game was localised to Finnish language by Turku University of Applied Sciences [15].

During the study the field tests were conducted in Turku, Finland, by Turku University of Applied Sciences in December 2015, and in Sendai, Japan, by Sendai City Industrial Promotion Organization (Sendai-Finland Wellbeing Center, Business Innovation International Unit) in February 2016. The field tests consisted of three different test setups, with the first one utilizing a near infra-red spectroscopy (NIRS) device to monitor the brain activity level of the test subject during the gameplay. The second test setup involved the test subjects playing the game in three groups of 4-5 people, and the last test setup allowed the test subjects to play the game individually. Within the second and third 
test setup the usability and acceptability of the Brain Trainer Exercise Game was evaluated with questionnaires and interviews developed by Sendai Television Broadcasting Company. These questionnaires were translated into Finnish for the Finnish test groups. All the different test setups also involved direct observation of the test subjects. The tests were conducted according to the same exactly protocol in Finland and Japan [15].

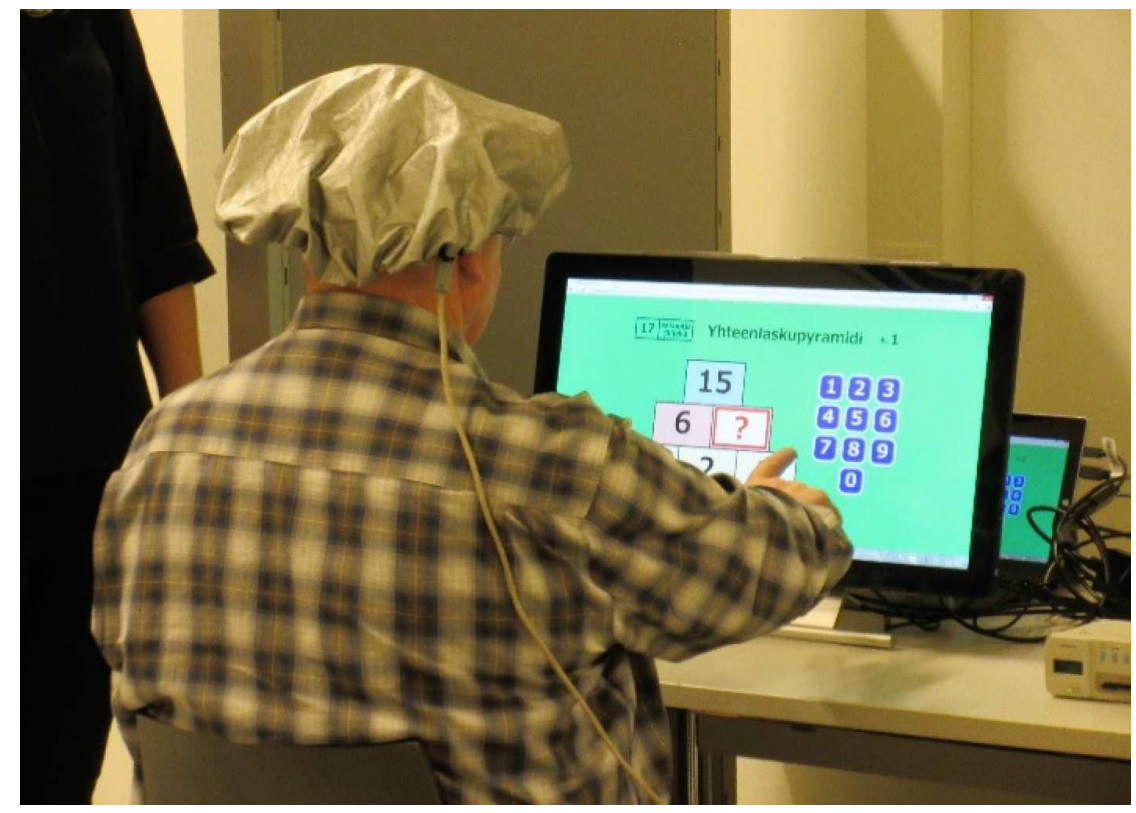

Figure 3 NIRS device being used for an elderly person form the Finnish test group.

The study employing the NIRS device as depicted in Figure 3 revealed that the elderly people within the test groups had a good and positive level of brain activity measured during the gameplay, both in the Japanese and in the Finnish test groups. This finding can lead to the conclusion that the brain trainer games may have a positive activating effect on healthy and non-diagnosed brains, which can furthermore act as one prevention method against cognitive problems, or slow down the natural ageing of the brain which can affect cognitive processes employing the working memory. The elderly people from the test groups from both countries were fluent enough with digital games and basic advice related to using the hardware devices and the game rules was sufficient. The test group in Finland had slightly more problems with the touch screen interface compared to the Japanese test group [15].

The hypothesis before the tests was that there would be more significant differences in the results from the two test groups, especially since the history of game industry and gamification is longer and more prominent in Japan compared to Finland. The quantitative data analysis did not however support the hypotheses and instead differences between the results was relatively low. The analysis of the test results was focused on the minigames which were most selected and played in both test groups, from all of the thirteen minigames. The tests revealed some cultural differences, for example the 
Japanese test subjects were reluctant to choose a game that might be too difficult for them, as they did not feel comfortable losing in the game in front of other people (the test arranging personnel and the other test subjects). The test subjects from the Finnish test group preferred to try out the different minigames in order, and in the test conditions they were reluctant to communicate before the gameplay if they considered the game to be difficult or not. Some test subjects might have commented the game "not being fun", and thus proceeding directly to the next minigame without having the desire to try out the more difficult levels of the same minigame [15].

One of the minigames displaying cultural differences between the two test groups was the "Rock, Scissors, Paper" - minigame, which has negated win condition for the game, therefore requiring the player to consciously attempt to lose in the game to provide the correct answer. The elderly people from the Japanese test group were familiar with the game, however they were not eager to play the negated win condition version of the game, because this would compel them to "lose" in this version of the game. A large portion of the test subjects from the Finnish test group were not familiar with the Rock, Scissors, Paper-game at all. Despite this they displayed eagerness to at least try the minigame out [15].

\subsection{Literature review on Motivational Factors for Elderly Stroke Patients}

We conducted a literature review on the motivational factors for stroke patients in the context of rehabilitation. Researchers advocate that playing games can have positive impacts and improve the quality of life of the elderly. Digital games can be used as an enhancement of mental and physical activities as well as a socialization tool for elderly. In addition, the motivational levels of the elderly stroke patients can be increased by using rehabilitation systems based on augmented reality. Based on the findings from the literature review, the researchers recommend game design guidelines for physical rehabilitation games for stroke patients $[16,17]$.

Researchers indicate that social functioning (e.g. social connection and contact) plays an important role in motivating patients to be engaged in their rehabilitative exercises. The relationship between patient and therapist is also an important factor. Setting a relevant personal goal is an important factor to motivate stroke patients in rehabilitation. The researchers also indicate that the rehabilitative setting and environment should be taken into consideration as an important motivational factor for patients. In stroke rehabilitation, it is important for patients to receive adequate information from the healthcare professionals (e.g. doctors, nurses, and therapists) so that they can engage in the rehabilitation process. Furthermore, physical tasks should be meaningful for patients to perform (e.g. Activities of daily living) so that they can feel engaged in these activities. The researchers also point out the importance of customization and personalization in stroke rehabilitation. There are other motivational factors for stroke patients recommended by the researchers such as positive feedback from therapists, music, and recreational activities $[16,17]$.

Based on the motivational factors, the researchers recommend game design principles. For social connection and functioning, multiplayer games can be designed to motivate stroke patients. A virtual therapist in digital games can be designed to promote the relationship between patients and therapists. Goal-oriented digital games can be achieved 
by designing effective game level design. The meaningful rehabilitative tasks can be achieved by designing real-world activities such as sports, gardening, cooking, and cycling. Game feedback, help system, and tutorials can be designed to provide effective information for stroke patients. In digital games, providing positive feedback to patients can improve their motivation in rehabilitation. The researchers also recommend other game design guidelines such as effective game music, recreational activities, and game context. These design guidelines can provide insights into designing effective digital games to motivate stroke patients $[16,17]$.

\section{Conclusion}

In this paper, we have presented the results of testing various rehabilitative games for elderly in Finland and Japan. All the tests were part of the GSH project where gamified services for promoting exercise and enhancing quality of life are researched and developed for elderly people. First, we presented the results of the Japanese usability evaluation of a prototype exergame called "The Glider". Then, we explained the process and the results of testing SportWall game and two commercial physical activity games with Finnish elderly participants. We also presented our findings from the literature review and interviews on potential users on existing games for seniors. Next, we described the need for enhancing mental health and preventing memory disorders by proposing the "Old Photos on Map" web application where users can upload and view old photos. Furthermore, we presented the results of the usability testing of the exergames SportWall and PhysioWall that was conducted in Finland. We also suggested design guidelines for usability practitioners according to our observations from testing the Skiing Game in Japan. Then we describe the experiments conducted for the Brain Trainer Exercise game in Finland and Japan. Finally, we presented the findings of our research on motivational factors for the elderly and we recommended game design principles. We summarize the amount, age and country of the participants in each test case in Table 1.

According to all of our tests we can say that the elderly enjoy playing exergames, and we conclude that digital games can be an effective way to enhance the quality of life of the elderly. In general, the elderly gave positive feedback for the idea of using digital activity games for exercising. Especially the Skiing game was liked a lot. However, we need to take into consideration motivational, usability and safety factors when designing rehabilitative games for the elderly. In the case of "The Glider" we observed that the players where motivated while playing but motivation should also be maintained throughout the gameplay. Moreover, the elderly prefer control-free devices such as Kinect when they play. The game content and context should be simple and uncluttered. Also, the motion controls in the gameplay should be simple and jumping should be avoided for safety reasons. When talking about web applications such as "Old Photos on Map", the elements of the user interface should be simple also large text is preferred. In addition, the elderly should receive adequate information before starting to play. Furthermore, unfamiliar activities in the game concepts should be avoided. 
Table 1. Test participants in each test case

\begin{tabular}{|c|c|c|c|}
\hline Case & Participants & Age (years) & Country \\
\hline The Glider & 12 & $18-23$ & Japan \\
\hline $\begin{array}{c}\text { SportWall \& } 2 \\
\text { commercial games }\end{array}$ & 5 & $65-85$ & Finland \\
\hline $\begin{array}{l}\text { Old Photos on } \\
\text { Map }\end{array}$ & 45 & $30-93$ & Finland \\
\hline $\begin{array}{l}\text { SportWall \& } \\
\text { PhysioWall }\end{array}$ & 19 & $61-83$ & Finland \\
\hline Kinect & 8 & $64-78$ & Finland \\
\hline $\begin{array}{l}\text { Brain Trainer } \\
\text { game }\end{array}$ & 46 & $>60$ & Finland \\
\hline $\begin{array}{c}\text { Brain Trainer } \\
\text { game }\end{array}$ & 46 & $>60$ & Japan \\
\hline Skiing game & 24 & $60-85$ & Japan \\
\hline Skiing game & 21 & $60-85$ & Finland \\
\hline RecReha & 10 & $71-78$ & Finland \\
\hline $\begin{array}{l}\text { Experiments in } \\
\text { Singapore }\end{array}$ & 35 & $>65$ & Singapore \\
\hline
\end{tabular}

The arranging of the field tests is challenging. Getting suitable elderly for testing physical activity games was not easy. From arranging the field tests we have also learned that more playing and less time filling questionnaires would be more beneficial for this kind of research, suggesting for future research projects that more automated means of data gathering and analytics would bring added value to both the test arranging personnel and the test subjects. 
In the future, we intend to publish the results of the following experiments. In winter 2016, we tested the Japanese RecReha game in Finland. The participants of the experiments were 10 Finnish seniors who played the game individually and in groups of 5 people. The game utilizes Kinect and the concept of the game is to try to hit virtual balls doing different kinds of movements. In spring 2016, we collaborated with Nahyang Technological University in Singapore and conducted two usability studies which include both Finnish and Singaporean game-based physical exercises. In the first study, called "Gamified solutions in healthcare and rehabilitation. Pilot study" we used five different exergames including the Finnish Skiing game and Hiking game as well as the Singaporean Chinatown game and Japanese RecReha game. The main objective of this study is to understand the usability and the user experiences of the elderly in playing five different exergames. In the second study, called "Gamified solutions in healthcare and rehabilitation - special interest in therapeutic exercise" we conducted a six week intervention study where the aim was to investigate the differences between three groups that used different exercise methods. Each group had 10 participants. The first group exercised using exergames, the second group did conventional exercise and the last group continued their life normally.

\section{Acknowledgement}

We would like to thank all of our collaborators and partners in the GSH project. This work was supported by the Finnish Funding Agency for Technology and Innovation (Tekes), City of Turku, and several industrial partners. We would also like to thank all those who participated in the game development and testing including the elderly participants and the staff from the service homes.

\section{References}

[1] R. Raitoharju, M. Luimula, A. Pyae, P. Pitkäkangas and J. Smed: Serious Games and Active Healthy Ageing: A Pre-study. in Proceedings of the 5th International Conference Well-being in the Information Society, 2014

DOI: $10.1007 / 978-3-319-10211-5 \_16$

[2] United Nations, Department of International Economic and Social Affairs, Population Division. Concise report on the world population situation 2014, ST/ESA/SER.A/354. ISBN 978-92-1-151518-3., New York, 2014

[3] E. Orsega-Smith, J. Davis, K. Kelley Slavish and L. Gimbutas: Wii Fit Balance Intervention in Community Dwelling Older Adults. in Games for Health Journal, 2012

DOI: $10.1089 / \mathrm{g} 4 \mathrm{~h} .2012 .0043$

[4] W. R. Boot, M. Champion, D. P. Blakely, T. Wright, D. J. Souders and N. Charness: Video games as a means to reduce age-related cognitive decline attitudes, compliance, and effectiveness. Frontiers in Psychology, 2013 DOI: $10.3389 /$ fpsyg.2013.00031

[5] J. A. Anguera, J. Boccanfuso, J. L. Rintoul, O. Al-Hashimi, F. Faraji, J. Janowich, E. Kong, Y. Larraburo, C. Rolle, E. Johnston and A. Gazzaley: Video game training enhances cognitive control in older adults. Nature, 2013 DOI: $10.1038 /$ nature 12486 
[6] S. Kühn, T. Gleich, R. C. Lorenz, U. Lindenberger and J. Gallinat: Playing Super Mario induces structural brain plasticity gray matter changes resulting from training with a commercial video game. in Journal of Molecular Psychiatry, 2014 DOI: $10.1038 / \mathrm{mp} .2013 .120$

[7] A. Nakai, M. Luimula, S. Hongo and H. Vuola: Evaluating a Game Motion-Based Control by Using Kansei Engineering Knowledge. in Proceedings of the 3rd IEEE Conference on Cognitive Infocommunications, pp. 139-144, 2013

DOI: 10.1109/CogInfoCom.2013.6719229

[8] A. Nakai, A. Pyae, M. Luimula, S. Hongo, H. Vuola and J. Smed: Investigating the Effects of Motion-based Kinect Game System on the User's Cognition. in International Journal on Multimodal User Interfaces, pp. 403-411, 2015 DOI: $10.1007 / \mathrm{s} 12193-015-0197-0$

[9] A. Pyae, M. Luimula and J. Smed, "Investigating the Usability of Interactive Physical Activity Games for Elderly: A Pilot Study,” CogInfoCom 2015 - 6th IEEE International Conference on Cognitive Infocommunications, pp. 185-194, 2015

DOI: 10.1109/CogInfoCom.2015.7390588

[10] A. Pyae, R. Raitoharju, M. Luimula, P. Pitkäkangas and J. Smed: Serious Games and Active Healthy Ageing: A Pilot Usability Testing of Existing Games. in International Journal of Networking and Virtual Organisations, 18p, 2016 DOI: http://dx.doi.org/10.1504/IJNVO.2016.075129

[11] A. Yoshii, H. Malmivirta, M. Luimula, P. Pitkäkangas and T. Nakajima: Designing a Map-Based Application and a Conversational Agent for Addressing Memory Problems. in Proceedings of the 17th International Conference on Human-Computer Interaction, 2015

DOI: 10.1007/978-3-319-21380-4_58

[12] H. Malmivirta and S. Kivelä, "Yellow Cottage and a Patch of Potato," in Art and Culture - Keys for better Brain Health. Course Material from Turku University of Applied Sciences 102, 2015, pp. 26-93

[13] T.N. Liukkonen, T. Mäkilä, H. Ahtosalo, T. Heinonen, R. Raitoharju and P. Pitkäkangas: Perceptions of the Elderly Users of Motion Tracking Exergames

[14] A. Pyae, M. Luimula, T. Saarenpää, P. Granholm and J. Smed: When the Japanese Elderly Play a Finnish Physical Exercise Game: A Usability Study. in Journal of Usability Studies, 22p (accepted)

[15] N. Katajapuu, P. Granholm, M. Hiramatsu, E. Ishihara, J. Hirayama, P. Pitkäkangas, P. Qvist and M. Luimula: Brain trainer exercise game. Field tests in Finland and Japan. in Proceedings of the International Journal of Chemistry and Chemical Engineering Systems, pp. 39-45, 2016

[16] A. Pyae, M. Luimula and J. Smed: Understanding Stroke Patients' Motivation for Motivation-Driven Rehabilitative Game Design. in Proceedings of the International Conference on Pervasive Games, pp. 99-111, 2014 DOI: $10.1007 / 978-3-319-19656-5$ _16

[17] A. Pyae, M. Luimula and J. Smed: Rehabilitative Games for Stroke Patients. EAI Endorsed Transactions on Pervasive Games, Vol. 1/4, 11 p, 2015 DOI: $104106 /$ sg.1.4.e2 